\title{
Experimental and Theoretical Characterization of an AC Electroosmotic Micromixer
}

\author{
Naoki SASAKI, ${ }^{\dagger}$ Takehiko KITAMORI, and Haeng-Boo KIM \\ Department of Applied Chemistry, Graduate School of Engineering, The University of Tokyo, \\ 7-3-1 Hongo, Bunkyo, Tokyo 113-8656, Japan
}

\begin{abstract}
We have reported on a novel microfluidic mixer based on AC electroosmosis. To elucidate the mixer characteristics, we performed detailed measurements of mixing under various experimental conditions including applied voltage, frequency and solution viscosity. The results are discussed through comparison with results obtained from a theoretical model of AC electroosmosis. As predicted from the theoretical model, we found that a larger voltage $\left(\sim 20 \mathrm{~V}_{\mathrm{p}-\mathrm{p}}\right)$ led to more rapid mixing, while the dependence of the mixing on frequency $(1-5 \mathrm{kHz})$ was insignificant under the present experimental conditions. Furthermore, the dependence of the mixing on viscosity was successfully explained by the theoretical model, and the applicability of the mixer in viscous solution $(2.83 \mathrm{mPa} \mathrm{s})$ was confirmed experimentally. By using these results, it is possible to estimate the mixing performance under given conditions. These estimations can provide guidelines for using the mixer in microfluidic chemical analysis.
\end{abstract}

(Received April 24, 2010; Accepted May 30, 2010; Published July 10, 2010)

\section{Introduction}

Over the past decade, there has been increased interest in the development of micro total analysis systems employing the characteristics of microfluids and small spaces. ${ }^{1}$ In microfluidic devices, the merging of two fluid streams into one stream in a Y-shaped microchannel is a typical operation employed to control chemical processes. Due to the formation of a stable laminar flow in the microchannel, the merged stream adopts a parallel two-layered flow even if the streams are miscible. Rapid mixing of solutes can take place without an externally applied force for the mixing since the mass-transfer distances in the microchannels are of micrometer order. It should be noted that the mixing occurs not by mixing of the fluids themselves, but by diffusion of the solutes across the streams (diffusional mixing). Therefore, the mixing time is governed by the diffusion coefficient of the solutes, and the mixing time for a large molecule having a small diffusion coefficient will be long. In such cases, an additional force or mechanism is necessary to accelerate the mixing. In fact, a number of micromixers have been reported to control mixing in a microchannel, and include passive and active mixers utilizing the energy of flow itself and externally applied energy for mixing, respectively.,2,3

In a previous paper, ${ }^{4}$ we reported on the development of a novel active micromixer utilizing $\mathrm{AC}$ electroosmotic flow (AC-EOF) in a microchannel. AC-EOF represents the fluid

$\dagger$ To whom correspondence should be addressed.

E-mail: sasakina@fc.jwu.ac.jp

N. S. present address: Department of Chemical and Biological Sciences, Faculty of Science, Japan Women's University, 2-8-1 Mejirodai, Bunkyo, Tokyo 112-8681, Japan.

H.-B. K. present address: Faculty of Science, Ibaraki University, 2-1-1 Bunkyo, Mito, Ibaraki 310-8512, Japan. flow generated by interaction between the tangential electric field and the charge on a pair of coplanar electrodes. We arranged a pair of electrodes having a sinusoidal inter-electrode gap in parallel to the channel as shown in Fig. 1. Such an electrode arrangement could induce meandering screwed-flow in the microchannel for efficient fluid mixing. As a result, the AC-EOF mixer successfully reduced the mixing time to $\sim 1 / 20$ that of diffusional mixing. ${ }^{4}$ Similar mixers were subsequently reported by others. ${ }^{5-7}$ These experimental results obtained with our mixer and by others clearly indicate the applicability of $\mathrm{AC}-\mathrm{EOF}$ to fluid mixing in microfluidic devices.

In addition to these experimental studies, theoretical treatments of AC-EOF have also progressed. Just as in the case of electroosmosis in a DC electric field, surface charge density and electric field are important factors in determining the velocity of AC-EOF. From analyses of these factors and the

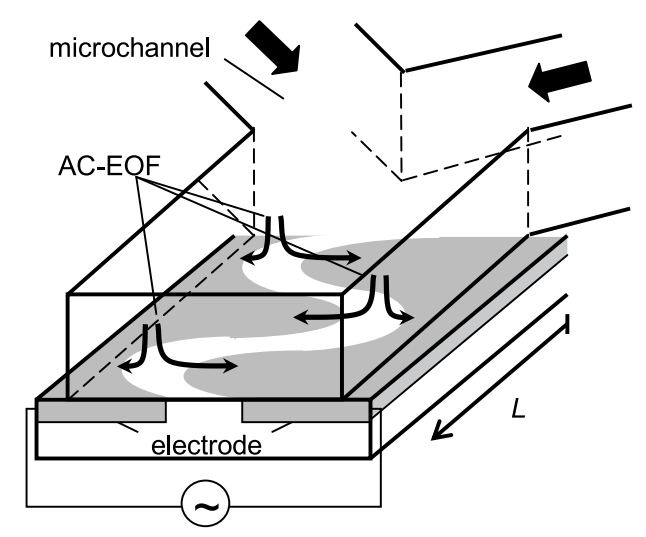

Fig. 1 Schematic illustration of AC-EOF micromixer. 
Helmholtz-Smoluchowski equation, the velocity of AC-EOF on an electrode has been discussed. Castellanos et al. ${ }^{8}$ reported an analytical equation for the time-averaged velocity of AC-EOF on an electrode $(\langle v\rangle)$ in two-dimensional form;

$$
\langle v\rangle=\Lambda \cdot \frac{\varepsilon V_{0}^{2}}{8 \eta x} \cdot \frac{\Omega^{2}}{\left(1+\Omega^{2}\right)^{2}}
$$

where $\varepsilon$ and $\eta$ represent the permittivity and viscosity of the solution, respectively. $V_{0}$ and $x$ are the amplitude of the applied voltage and the distance from the center of the inter-electrode gap, respectively. The dimensionless frequency, $\Omega$, is defined as;

$$
\Omega=\Lambda \frac{\omega \varepsilon \pi x}{2 \sigma \lambda_{\mathrm{D}}}
$$

where $\omega, \sigma$, and $\lambda_{\mathrm{D}}$ represent the angular frequency of the applied voltage, conductivity of the solution, and Debye length, respectively. $\Lambda$ is the ratio of the potential drop across the diffuse electric double layer $\left(\Delta \phi_{\mathrm{d}}\right)$ to that across the entire double layer $\left(\Delta \phi_{\mathrm{DL}}\right)$, which can be rewritten using the Stern layer capacitance $\left(C_{\mathrm{s}}\right)$ and the diffuse electric double layer capacitance $\left(C_{\mathrm{d}}\right)$.

$$
\Lambda=\frac{\Delta \phi_{\mathrm{d}}}{\Delta \phi_{\mathrm{DL}}}=\frac{C_{\mathrm{s}}}{C_{\mathrm{s}}+C_{\mathrm{d}}}
$$

The validity of Eq. (1) has been confirmed in dilute aqueous solution of salt with low conductivity $\left(\sim 8.6 \mathrm{mS} \mathrm{m}^{-1}\right)$ and with small applied voltage $\left(\sim 1 \mathrm{~V}_{\mathrm{p}-\mathrm{p}}\right) .^{9}$

The mixing efficiency of the AC-EOF mixer is principally governed by the velocity of AC-EOF. Although the flow profile in the AC-EOF mixer is complicated (meandering screwed-flow; see electronic supplementary information in the previous paper $)^{4}$ and the calculation of the velocity in the mixer is difficult, we can expect application of Eq. (1) to provide useful information concerning the characteristics of the AC-EOF mixer. According to Eq. (1), factors determining $\langle v\rangle$ are roughly categorized as the solution properties and terms related to the applied voltage ( $V_{0}$ and $\omega)$. Even if direct comparison between $\langle v\rangle$ and the mixing efficiency of the AC-EOF mixer is difficult, it is necessary to study the dependence of the mixing efficiency on the experimental conditions in an effort to elucidate the characteristics of the mixer. In this paper, we present the effects of applied voltage and solution property on the AC-EOF mixer. Our results revealed that the mixing efficiency of the mixer can be successfully explained by the theoretical model of AC-EOF, and that such an explanation can be utilized to predict the performance of the mixer under given conditions.

\section{Experimental}

The AC-EOF mixer employed in this study, which comprised a Y-shaped microchannel with a pair of coplanar electrodes having a sinusoidal gap, was the same device utilized as previously reported. ${ }^{4}$ The channel width and depth were 120 and $40 \mu \mathrm{m}$, respectively. The gap between the electrodes and the periodicity were 40 and $250 \mu \mathrm{m}$, respectively. The electrodes were positioned $0.4 \mathrm{~mm}$ downstream from the confluence of the Y-shaped channel.

Conventional dilution experiments were conducted using fluorescein (Tokyo Kasei) as the fluorescent dye to visualize the mixing process. ${ }^{4}$ Two aqueous solutions were used, one dyed $\left(2 \times 10^{-5} \mathrm{~mol} \mathrm{dm}^{-3}\right.$ fluorescein $)$ and the other not dyed

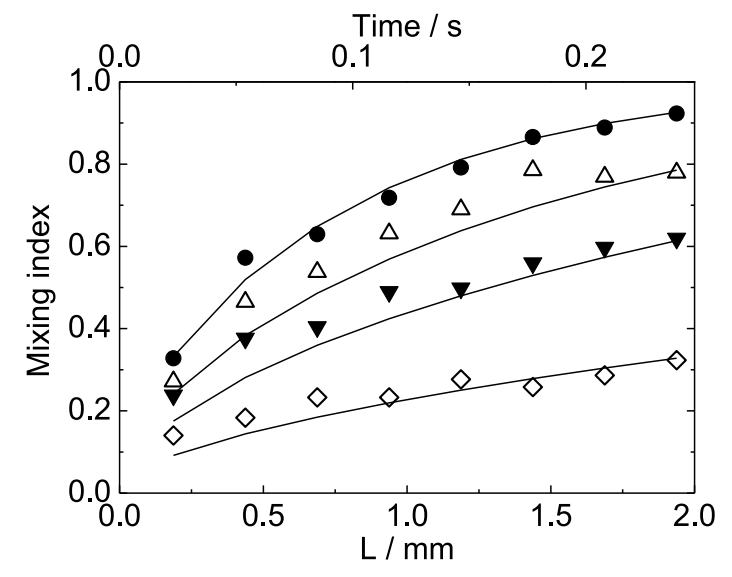

Fig. 2 Variation of the mixing index along the microchannel with different applied voltage: $20 \mathrm{~V}_{\mathrm{p}-\mathrm{p}}$ (filled circles), $16 \mathrm{~V}_{\mathrm{p}-\mathrm{p}}$ (open triangles), $12 \mathrm{~V}_{\mathrm{p}-\mathrm{p}}$ (filled inverted triangles) and $0 \mathrm{~V}_{\mathrm{p}-\mathrm{p}}$ (open diamonds, diffusional mixing). The frequency was $1 \mathrm{kHz}$. The value of $L$ was converted to time (shown on the upper x-axis) by dividing $L$ by the mean velocity $\left(8.1 \mathrm{~mm} \mathrm{~s}^{-1}\right)$. The solid lines represent the calculated mixing index obtained using the apparent diffusion coefficients.

( $2 \times 10^{-5} \mathrm{~mol} \mathrm{dm}^{-3} \mathrm{KNO}_{3}$, Wako Pure Chemical Industries). The viscosity of each solution was adjusted with ethylene glycol (Aldrich). The solutions were bubbled with Ar for $30 \mathrm{~min}$ to avoid possible degradation of the dye by electroactivated oxygen in the solution, and then introduced into the Y-shaped channel using a two-port syringe pump (Model 210, KD Scientific). In the present study, the flow rate of each solution was set at $1 \mu \mathrm{L} \mathrm{min}{ }^{-1}$. From the flow rate and the cross-sectional area of the channel, the mean velocity in the mixing channel was calculated to be $8.1 \mathrm{~mm} \mathrm{~s}^{-1}$. The applied voltage $\left(0-20 \mathrm{~V}_{\mathrm{p}-\mathrm{p}}\right)$ and frequency $(\sim 5 \mathrm{kHz})$ were controlled using a PC equipped with a digital-to-analog converter (DAQCard-6024E, National Instruments). The mixing processes were visualized using a fluorescence microscope (SZX12, Olympus) equipped with a CCD camera (DXC-200A, Sony). The video images were recorded with a graphic capture card (GV/MPEG2-PCI, I-O DATA). After correcting fluorescence intensity to remove optical effects of the electrodes, intensity profiles were obtained. The mixing index was calculated from the standard deviation of the lateral intensity profile at a given position ( $L$ in Fig. 1$)$. The initial (not mixed) and fully mixed mixing index was assigned as 0 and 1 , respectively. Mixing length $\left(X_{90}\right)$ was determined as $L$ where the mixing index was 0.9 . Since a linear dependence was observed between $X_{90}$ and the flow velocity, the mixing time $\left(T_{\text {mix }}\right)$ was defined as the slope of the regression line.

\section{Results}

Figure 2 shows the dependence of the mixing index on distance ( $L$ in Fig. 1). In the absence of an applied voltage (diffusional mixing), the mixing index increased with increasing $L$, and was 0.33 at $L=1.94 \mathrm{~mm}$. An increase in the voltage clearly accelerated the mixing as shown in Fig. 2. With an applied voltage of $20 \mathrm{~V}_{\mathrm{p}-\mathrm{p}}$, the mixing index increased to 0.92 and resulted in almost complete mixing at $L=1.94 \mathrm{~mm}(0.24 \mathrm{~s})$. As previously reported, ${ }^{4}$ the $T_{\text {mix }}$ in diffusional mixing is estimated to be $4.4 \mathrm{~s}$ from the diffusion coefficient of the dye. Therefore, voltage application clearly reduced the mixing time compared to diffusional mixing. In the following, we discuss the mixing 


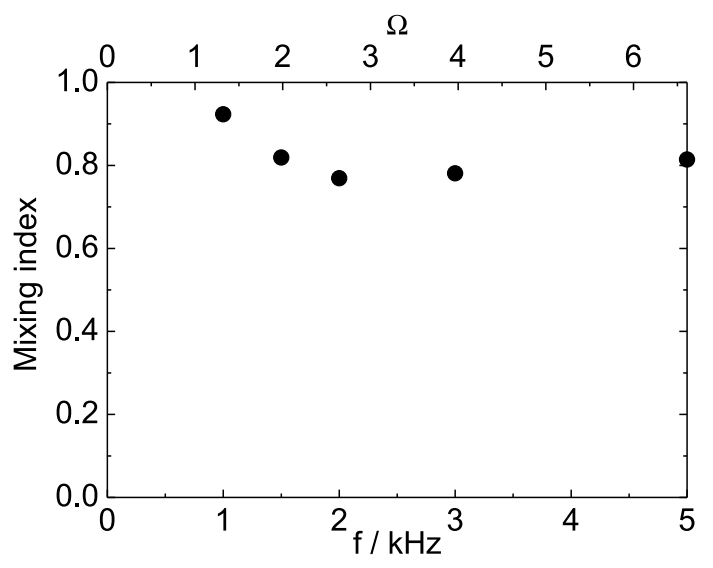

Fig. 3 Dependence of the mixing index on frequency. The applied voltage was $20 \mathrm{~V}_{\mathrm{p}-\mathrm{p}}$.

based on the mixing index obtained at $L=1.94 \mathrm{~mm}$.

The dependence of the mixing index on frequency at fixed amplitude $\left(20 \mathrm{~V}_{\mathrm{p}-\mathrm{p}}\right)$ is shown in Fig. 3. The mixing index decreased slightly with increasing frequency from 1 to $5 \mathrm{kHz}$. These experimental results are consistent with theoretical prediction of AC-EOF in Eq. (1), in which $\langle v\rangle$ reaches its maximum value when $\Omega=1$ and decreases with increasing (or decreasing) $\Omega$. Below $1 \mathrm{kHz}$, a decrease in total fluorescence intensity was observed, probably due to the $\mathrm{pH}$ change caused by the electrolysis of water. Therefore, data were taken at greater than $1 \mathrm{kHz}$. Under the present experimental conditions, we achieved the maximum mixing index of 0.92 at an applied voltage of $20 \mathrm{~V}_{\mathrm{p}-\mathrm{p}}$ and $1 \mathrm{kHz}$.

In an effort to investigate the dependence of the AC-EOF mixer on viscosity, ethylene glycol was added to the water up to $40 \mathrm{wt} \%$. The addition of ethylene glycol is known to increase $\eta$ from 1.00 to $2.83 \mathrm{mPa}$ s at $40 \mathrm{wt} \% .^{10}$ Although $\varepsilon$ and $\sigma$ vary with the addition of ethylene glycol, the changes are relatively small compared to the change in $\eta$. The $\varepsilon$ values of water and $40 \mathrm{wt} \%$ aqueous ethylene glycol solution were $7.12 \times 10^{-10}$ and $6.06 \times 10^{-10} \mathrm{~F} \mathrm{~m}^{-1}$, respectively. ${ }^{11}$ The $\sigma$ values of the test solution in the absence or presence of $40 \mathrm{wt} \%$ ethylene glycol were $3.91 \times 10^{-4}$ and $2.76 \times 10^{-4} \mathrm{~S} \mathrm{~m}^{-1}$, respectively. Therefore, the addition of ethylene glycol mainly affects the viscosity, thereby allowing for a discussion concerning the dependence of the mixing on viscosity.

The dependence of the mixing index on viscosity is shown in Fig. 4. The results clearly indicated that the mixing index decreased with increasing viscosity. In $40 \mathrm{wt} \%$ aqueous ethylene glycol solution, the mixing index was almost half that in water. This decrease in mixing index corresponded to an increase in the mixing time. $T_{\text {mix }}$ values in water and $40 \mathrm{wt} \%$ aqueous ethylene glycol solution were 0.19 and $0.63 \mathrm{~s}$, respectively (data not shown). However, the rate of mass-transfer by diffusion decreases with increasing viscosity, so that it is necessary to compare the results with that of diffusional mixing. First, we calculated the diffusion coefficients at various $\eta$ using the Stokes-Einstein equation. These values were then used to estimate the mixing index in the viscous solution according to the method described later. The estimated results plotted in Fig. 4 agree with the experimental values for diffusional mixing. The mixing index by diffusional mixing in $40 \mathrm{wt} \%$ solution was 0.13 . $T_{\text {mix }}$ was estimated to be $12.5 \mathrm{~s}$, which was 20 -fold longer than that of diffusional mixing $(0.63 \mathrm{~s})$. The ratio of $T_{\text {mix }}$ for diffusional mixing to that for the present $\mathrm{AC}$-EOF mixer in

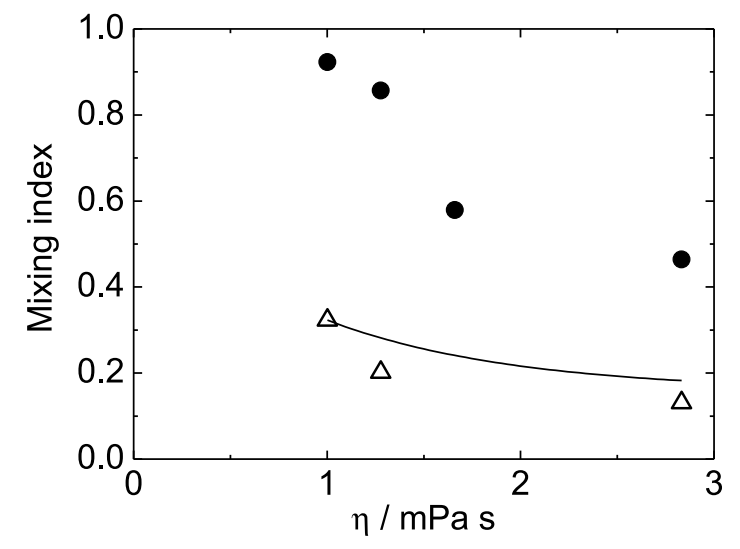

Fig. 4 Dependence of the mixing index on viscosity obtained experimentally for the present AC-EOF mixer (filled circles) and for diffusional mixing (open triangles). The solid line represents the calculated mixing index for diffusional mixing using the diffusion coefficients calculated by the Stokes-Einstein equation. The applied voltage and frequency were $20 \mathrm{~V}_{\mathrm{p}-\mathrm{p}}$ and $1 \mathrm{kHz}$, respectively.

$40 \mathrm{wt} \%$ solution was almost the same as the ratio in water $(\sim 20)$. It is clear that the AC-EOF mixer worked well even in the viscous solution.

\section{Discussion}

As shown above, the performance of the AC-EOF mixer depended on various experimental conditions. To discuss the effect of AC-EOF on the present mixing, we have to isolate the effect of diffusional mixing since mixing is induced by both diffusional and AC-EOF mixing effects. The difference in the obtained mixing index with and without voltage application can be considered as an index representing the AC-EOF effect alone. Although the difference reflects the mass-transfer by AC-EOF, it is difficult to relate the difference to the velocity of AC-EOF directly. As an alternative approach, we consider the mixing process in the mixer as being similar to diffusional mixing and assume that AC-EOF causes an apparent increase in the diffusion coefficient. This assumption implies that the apparent diffusion coefficient $\left(D_{\text {mix }}\right)$ can be used as an index of the mass-transfer for mixing and the mixing index can be calculated based on Fick's law. To calculate the mixing index, the concentration distribution of solutes in a microchannel was determined using the two-dimensional analytical equation: ${ }^{12}$

$$
\begin{aligned}
c^{*}\left(x^{*}, y^{*}\right)=\frac{1}{\pi} \sum_{\mathrm{n}=1}^{\infty}\left[\exp \left[\frac{-\pi^{2}(2 n-1)^{2} x^{*}}{P e}\right] \times\right. \\
\left.\sin \left[\pi(2 n-1) y^{*}\right] \frac{1-\cos [\pi(2 n-1)]}{2 n-1}\right]
\end{aligned}
$$

$P e=u W / D$

where $c^{*}$ is the dimensionless concentration, $x^{*}$ and $y^{*}$ are the dimensionless position along and across a channel, respectively, $P e$ is the Peclet number, $u$ is the fluid velocity along the channel, $W$ is the channel width, and $D$ is the diffusion coefficient of solute. The mixing index at $x^{*}$ was then calculated from the standard deviation of $c^{*}$. The above calculations were repeated while changing the $D$ value until the calculated mixing index at 


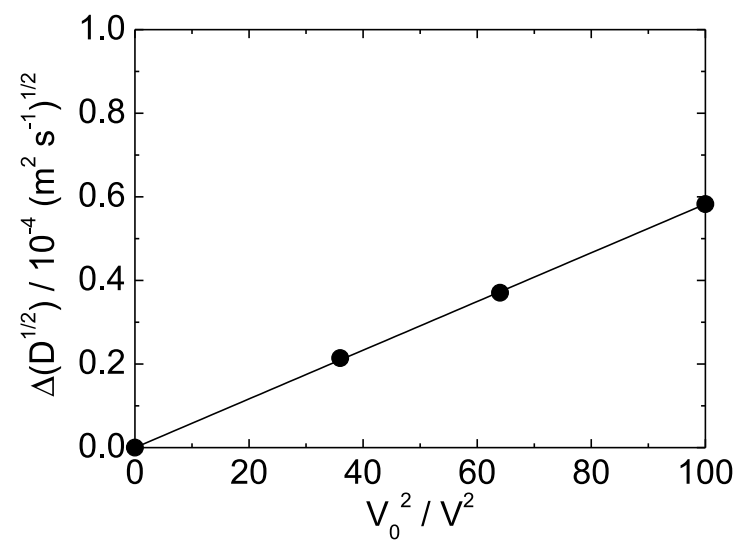

Fig. 5 Variation of $\Delta\left(D^{1 / 2}\right)$ with the square of the amplitude obtained from data at $L=1.94 \mathrm{~mm}$ in Fig. 2. The solid line represents the regression line obtained assuming a proportional relationship between the parameters.

$L=1.94 \mathrm{~mm} \quad\left(x^{*}=16.2\right)$ agreed with the experimentally determined index, and $D_{\text {mix }}$ under a given condition was determined. To verify the validity of this approach, the dependence of the mixing index on $L$ for each voltage was simulated by the obtained $D_{\text {mix }}$ values. The results (Fig. 2, solid lines) successfully reproduced the experimentally determined values, thereby allowing for a discussion of the mixing based on $D_{\text {mix }}$.

From the $D_{\text {mix }}$ values obtained by the above method, the effect of AC-EOF on mixing can be estimated as follows. Assuming that the apparent mixing rate $\left(N_{\text {mix }}\right)$ represents the sum of the mass-transfer rate across the channel by AC-EOF $\left(N_{\mathrm{EO}}\right)$ and by diffusion $\left(N_{\mathrm{D}}\right)$,

$$
N_{\text {mix }}=N_{\text {EO }}+N_{\text {D }}
$$

$N_{\mathrm{D}}$ can be related to the diffusion coefficient of solute $\left(D_{0}\right)$;

$$
N_{\mathrm{D}}=A\left(2 D_{0} / t\right)^{1 / 2}
$$

where $t$ is the time from the beginning of the mixing and $A$ is a constant. Similarly, $N_{\text {mix }}$ can be related to $D_{\text {mix }}$,

$$
N_{\text {mix }}=A\left(2 D_{\text {mix }} / t\right)^{1 / 2}
$$

From Eqs. (6) - (8),

$$
N_{\text {EO }} \propto D_{\text {mix }}{ }^{1 / 2}-D_{0}^{1 / 2} \equiv \Delta\left(D^{1 / 2}\right)
$$

Thus, it is possible to extract the contribution of AC-EOF to the mixing from the experimentally obtained mixing index as $\Delta\left(D^{1 / 2}\right)$, which allows for a discussion of the mixing based on AC-EOF velocity $\langle v\rangle$, shown in Eq. (1).

Figure 5 shows the dependence of $\Delta\left(D^{1 / 2}\right)$ on amplitude, in which the data were plotted against $V_{0}^{2}$ according to Eq. (1). The regression line is also shown in the figure. A good correlation between $\Delta\left(D^{1 / 2}\right)$ and $V_{0}^{2}$ was found. This result suggests that $N_{\mathrm{EO}}$ was appropriately evaluated using $\Delta\left(D^{1 / 2}\right)$. In other words, the efficiency of the AC-EOF mixer can be described by the theoretical model in terms of amplitude, even though the voltage applied in the present experiments $\left(20 \mathrm{~V}_{\mathrm{p}-\mathrm{p}}\right)$ was much larger than values shown to follow Eq. (1) $\left.\left(\sim 1 \mathrm{~V}_{\mathrm{p}-\mathrm{p}}\right)\right)^{9}$

Compared to the amplitude dependence, the dependence of

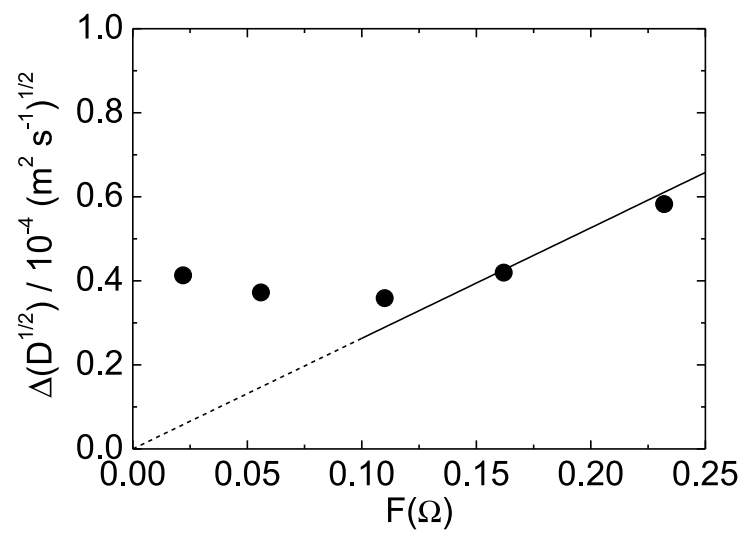

Fig. 6 Variation of $\Delta\left(D^{1 / 2}\right)$ with $F(\Omega)$ obtained from data in Fig. 3. The solid line represents the regression line at $F(\Omega)>0.1$ obtained assuming a proportional relationship between the parameters.

mass-transfer on frequency by AC-EOF is complicated. As can be seen in Eqs. (1) and (2), the frequency dependent term is $\Omega$, which affects $\langle v\rangle$ through the third term in Eq. (1); $\Omega^{2} /\left(1+\Omega^{2}\right)^{2}$. For simplicity, the third term was denoted as $F(\Omega)$ in the following. From the variation of $F(\Omega)$, Eq. (1) gives a bell-shaped profile for the frequency dependence of $\langle v\rangle$, which reaches its maximum value when $\Omega=1$. In fact, Green et al ${ }^{13}$ observed a maximum $\langle v\rangle$ in the frequency range of several hundred $\mathrm{Hz}$ to a few $\mathrm{kHz}$. As shown in Fig. 3, a bell-shaped dependence of the mixing index on frequency was not observed due to water electrolysis below $1 \mathrm{kHz}$, although there was a tendency for the mixing index to decrease with increasing frequency. Figure 6 shows $\Delta\left(D^{1 / 2}\right)$ obtained at various frequencies plotted against $F(\Omega)$ calculated using the reported value of 0.25 for $\Lambda^{9}$ and the calculated value of $6.8 \times 10^{-8} \mathrm{~m}$ for $\lambda_{\mathrm{D}}$ from salt concentration. ${ }^{14}$ Although some discrepancies were observed with small $F(\Omega)$ (high frequency), a proportional relationship between $\Delta\left(D^{1 / 2}\right)$ and $F(\Omega)$ was apparent. A discrepancy at high frequency was also reported for the frequency dependence of $\langle v\rangle$, in which a spread of relaxation times associated with the geometry and electrode polarization was suggested. ${ }^{13}$ Therefore, the dependence of AC-EOF mixing on frequency could be roughly explained by the theoretical model. This result implies that the optimum frequency for AC-EOF mixing is $\sim 1 \mathrm{kHz}$, and that further acceleration of the mixing cannot be expected by changing the frequency.

Figure 7 shows the variation of $\Delta\left(D^{1 / 2}\right)$ with the inverse of the solution viscosity. As expected from Eq. (1), $\Delta\left(D^{1 / 2}\right)$ was proportional to $\eta^{-1}$. This result indicates that the solution viscosity works as a form of resistance to solute motion in the AC-EOF mixing process, similarly to cases involving diffusion or DC electroosmosis processes.

We demonstrated the characteristics of the AC-EOF micromixer through analysis of the effects of applied voltage and solution properties, which were successfully explained by the theoretical model for $\langle v\rangle$. It should be mentioned that the effect of conductivity of the solution is also to be examined in the analysis for a better understanding of the mixer. A change in salt concentration causes the changes in $\Lambda$ and $F(\Omega)$ through $C_{\mathrm{d}}, \sigma$ and $\lambda_{\mathrm{D}}$ as shown in Eqs. (1) - (3). With increasing salt concentration of solution, $\Lambda$ decreases due to increase in $C_{\mathrm{d}}$. However, it is difficult to estimate the values of $\Lambda$ for each salt concentration. The value of $C_{\mathrm{d}}$ depends on the potential at the outer Helmholtz plane in the Gouy-Chapman-Stern model, 


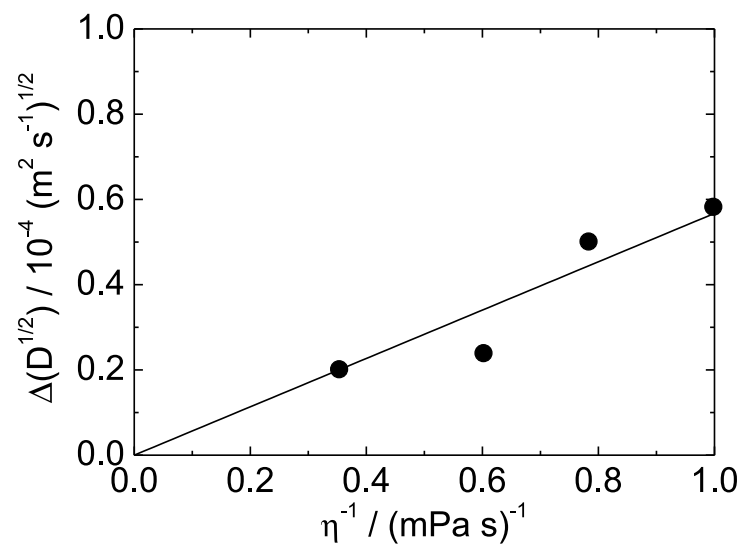

Fig. 7 Variation of $\Delta\left(D^{1 / 2}\right)$ with the inverse of the viscosity obtained from data in Fig. 4. The solid line represents the regression line assuming a proportional relationship between the parameters.

Table 1 Slopes of regression lines in Figs. 5-7 and $Q$ values calculated from Eq. (10)

\begin{tabular}{cccccc}
\hline \multirow{2}{*}{$\begin{array}{c}\text { Variable } \\
\text { parameter, }\end{array} \begin{array}{c}\text { Slope of } \\
P\end{array}$} & $\begin{array}{c}\text { regression } \\
\text { line, }\end{array}$ & \multicolumn{3}{c}{ Fixed parameter } & \\
\cline { 3 - 5 } & $\Delta\left(D^{1 / 2}\right) / P$ & $V_{0}^{2} / V^{2}$ & $F(\Omega)^{\mathrm{a}}$ & $\begin{array}{c}\eta^{-1} / \\
(\mathrm{mPa} \mathrm{s})^{-1}\end{array}$ & $Q / \mathrm{F} \mathrm{m} \mathrm{m}^{-2}$ \\
\hline$V_{0}^{2}$ & $5.83 \times 10^{-7}$ & - & 0.232 & 1.00 & $2.5 \times 10^{-6}$ \\
$F(\Omega)$ & $2.63 \times 10^{-4}$ & 100 & - & 1.00 & $2.6 \times 10^{-6}$ \\
$\eta^{-1}$ & $5.67 \times 10^{-5}$ & 100 & 0.232 & - & $2.4 \times 10^{-6}$ \\
\hline
\end{tabular}

a. $F(\Omega)$ value of 0.232 corresponds to $1 \mathrm{kHz}$.

which is the basis of the theoretical model of AC-EOF. ${ }^{8}$ The applied voltage in the present experiments is much higher than the voltage that was used for experimental investigation of $C_{\mathrm{s}}$ and $C_{\mathrm{d}}{ }^{15}$ Moreover, the change in $\Lambda$ leads to the change in $\Omega$ as shown in Eq. (2), which also makes it difficult to estimate the value of $F(\Omega)$. As mentioned by Green et al., ${ }^{9}$ more theoretical and experimental work is required to understand the dependence of AC-EOF and thus the AC-EOF mixing.

It should be emphasized that the performance of the mixer could be evaluated by the apparent diffusion coefficient $\left(D_{\text {mix }}\right)$. In fact, as expected from Eq. (1), we observed a proportional relationship between $\Delta\left(D^{1 / 2}\right)$ and $V_{0}^{2}, F(\Omega)$, or $\eta^{-1}$ as shown in Figs. 5 - 7. This suggests that $\Delta\left(D^{1 / 2}\right)$ can be described as

$$
\Delta\left(D^{1 / 2}\right)=Q \cdot V_{0}^{2} \cdot F(\Omega) \cdot \eta^{-1}
$$

where $Q$ is a proportional constant, and should take the same value irrespective of the variable parameters $\left(P: V_{0}^{2}\right.$ in Fig. 5, $F(\Omega)$ in Fig. 6, $\eta^{-1}$ in Fig. 7).
The slope in Figs. $5-7$ corresponds to $\Delta\left(D^{1 / 2}\right) / P$. The $Q$ values calculated using the appropriate fixed parameters are also summarized in Table 1 and agree well with each other. This result supports the validity of the assumption employed. Therefore, it is possible to calculate $\Delta\left(D^{1 / 2}\right)$ using the slopes, and then to estimate the mixing performance for a given condition as $D_{\text {mix }}$ from Eq. (9). Moreover, the mixing index can be estimated from the concentration profile calculated from Eqs. (4) and (5). These estimations can provide guidelines for using the AC-EOF mixer in microfluidic chemical analysis, such as the labeling of analytes prior to detection, or the preparation of various concentrations of solutions from a single standard solution.

\section{Acknowledgements}

The authors acknowledge Grants-in-Aid from the Murata Science Foundation for supporting part of the research.

\section{References}

1. J. West, M. Becker, S. Tombrink, and A. Manz, Anal. Chem., 2008, 80, 4403.

2. V. Hessel, H. Lowe, and F. Schonfeld, Chem. Eng. Sci., 2005, 60, 2479.

3. N. T. Nguyen and Z. G. Wu, J. Micromech. Microeng., 2005, 15, R1.

4. N. Sasaki, T. Kitamori, and H.-B. Kim, Lab Chip, 2006, 6, 550 .

5. S. H. Huang, S. K. Wang, H. S. Khoo, and F. G. Tseng, Sens. Actuators, B, 2007, 125, 326.

6. W. Y. Ng, S. Goh, Y. C. Lam, C. Yang, and I. Rodriguez, Lab Chip, 2009, 9, 802.

7. J. K. Chen, C. N. Weng, and R. J. Yang, Lab Chip, 2009, 9, 1267.

8. A. Castellanos, A. Ramos, A. Gonzalez, N. G. Green, and H. Morgan, J. Phys. D: Appl. Phys., 2003, 36, 2584.

9. N. G. Green, A. Ramos, A. Gonzalez, H. Morgan, and A. Castellanos, Phys. Rev. E, 2002, 66, 026305.

10. D. R. Lide, "CRC Handbook of Chemistry and Physics", 82nd ed., 2001, CRC Press, Boca Raton.

11. F. Franks, "Water", 1973, Vol. 2, Plenum Press, New York.

12. Z. G. Wu, N. T. Nguyen, and X. Y. Huang, J. Micromech. Microeng., 2004, 14, 604 .

13. N. G. Green, A. Ramos, A. Gonzalez, H. Morgan, and A. Castellanos, Phys. Rev. E, 2000, 61, 4011.

14. A. J. Bard and L. R. Faulkner, "Electrochemical Methods: Fundamentals and Applications", 2nd ed., 2001, John Wiley \& Sons, New York, 548.

15. D. C. Grahame, Chem. Rev., 1947, 41, 441. 